\title{
Effects of Polyphenolic Extract from Pine Bark on the Improvement of Attention Deficit/Hyperactivity Disorder in Children and Adolescent
} Ying-Ru Chen ${ }^{1}, Y u-J u$ Su$^{2}$, Yannick Piriou ${ }^{3}$, Suh-Ching Yang ${ }^{2,4^{*}}$

${ }^{1}$ Department of Psychiatry, Shuang Ho Hospital, Taipei Medical University, No. 291, Zhongzheng Rd., Zhonghe District, New Taipei City 235, Taiwan

${ }^{2}$ School of Nutrition and Health Sciences, College of Nutrition, Taipei Medical University, No. 250 Wu-Hsing Street, Taipei 11031, Taiwan

${ }^{3}$ Les Derives Resiniques\&Terpeniques, 40560 Vielle Saint Girons, France

${ }^{4}$ Research Center of Geriatric Nutrition, College of Nutrition, Taipei Medical University, No. 250 Wu-Hsing Street, Taipei 11031, Taiwan

\begin{abstract}
Purpose: To examine the hypothesis that intervention with the polyphenolic complex (PE) from pine bark improves the symptoms of ADHD and reduces oxidative stress in children and adolescent.

Methods: It was a randomized, double-blind, crossover and placebo-controlled 10-weeks period study, including two interventional periods (4weeks/period) and one washout period ( 2 weeks). Data were from 8 participants with attention deficit hyperactivity disorder (ADHD) at ages $7 \sim 16$ years (seven boys and one girl). During the first interventional period, participants were received a capsule of PE from pine bark, which contain $25 \mathrm{mg}$ Oligopin ${ }^{\oplus}$ per capsule, or a capsule of placebo, which contains $25 \mathrm{mg}$ cellulose. Then, participants entered the washout period for 2 weeks. After 2 weeks of washout, the participants entered the second interventional period and crossed over to receive an Oligopin ${ }^{\oplus}$ or placebo capsule. Neuropsychological assessment, routine blood biochemical parameter and antioxidative status were carried out in this study.

Results: All blood biochemical parameters were normal in the interventional periods. However, the lipid peroxidation was significantly decreased when participants received the PE capsules. Moreover, the participants had a significant higher hit accuracy, inhibition, sustainability in CPT-II performanceduring the PE interventional period.

Conclusions: The administration of the polyphenolic extract from pine bark ( $25 \mathrm{mg} /$ day $)$ for one month might improve the inattention and impulsivity and reduce plasma lipid peroxidation levels in children and adolescent with ADHD.
\end{abstract}

\section{Introduction}

Attention deficit hypertension disorder (ADHD) is one of the common neurodevelopment psychiatric disorders in children and adolescent, even in adults who are characterized by inattention, impulsivity and hyperactivity [1]. In Taiwan, an early community study in 1993 reported that the prevalence rates of ADHD was $19.8 \%$ for boys and $12.3 \%$ for girls among elementary students with a boy and girl ratio of 3:1[2]. However, comprehensive(nutritional) researches of ADHD in Taiwan is still rare.

At present, the real causes of ADHD are unknown, but it is thought to be biological and multifactorial, including genetic factors, the damage in catecholamine metabolism, lead toxicity, food sensitivities, or nutritional problems etc [3]. Recently, it is assumed that oxidative stress caused by the abnormal metabolism of adrenaline, noradrenaline and dopamine may play an important role in pathology of ADHD. Therefore, antioxidant supplements have been reported to replace the classical treatment of ADHD by psychostimulants and antidepressants because of the side effects of these psychomedicaments [4].

The bark extract of French maritime pine (Pinuspinaster) contains abundant polyphenolic compounds that are considered as the potent antioxidants. Heimann collected case reports about beneficial effects following treatment of polyphenolic extract (PE) from pine bark in French children with ADHD [5]. In our country, the active drugs use to ADHD patients is still controversial and the replacement therapy becomes the focal point, especially nutritional supplements. The purpose of this study was to investigate the improvement effects of $\mathrm{PE}$ from pine bark on the symptoms of ADHD and oxidative stress

\section{Publication History:}

Received: February 15, 2017

Accepted: May 17, 2017

Published: May 19, 2017

\section{Keywords:}

Polyphenolic extract from pine bark, Antioxidant, Attention deficit/hyperactivity disorder, Conners' Continuous Performance Test(CPT-II)

in children or adolescents. This is the first pilot study to discuss the effects of nutritional supplements on ADHD symptoms in Taiwan.

\section{Methods}

\section{Subjects}

The Clinical trials registry site of this study is TMU-JIRB (Taipei Medical University-Joint Institutional Review Board) and the TMU-JIRB number is 201404107. Participants were recruited from Psychiatric Clinic of the Shuang Ho hospital, Wanfang hospital, Taipei city hospital Songde branch and Grace counseling center, Taiwan. The inclusion criteria included participants who were 7 16 years old and met the definition of with attention deficit hyperactivity disorder (ADHD) as defined by SNAP-IV (Swanson, Nolan, and Pleham Version-IV). Then, in order to exclude the intelligence and other behavior problems, CPM (Clolured Progressive Matrices), SPM (Standard Progressive Matrices) and CBCL (Child Behavior Checklist) were used.

"Corresponding Author: Dr. Suh-Ching Yang, Research Center of Geriatric Nutrition, College of Nutrition, Taipei Medical University, No. 250 Wu-Hsing Street, Taipei 11031, Taiwan; E-mail: sokei@tmu.edu.tw

Citation: Chen YR, Su YJ, Piriou Y, Yang SC (2017) Effects of Polyphenolic Extract from Pine Bark on the Improvement of Attention Deficit/Hyperactivity Disorder in Children and Adolescent . Int J Clin Nutr Diet 3: 116. doi: https://doi. org/10.15344/2456-8171/2017/116

Copyright: (C) 2017 Chen et al. This is an open-access article distributed under the terms of the Creative Commons Attribution License, which permits unrestricted use, distribution, and reproduction in any medium, provided the original author and source are credited. 
Citation: Chen YR, Su YJ, Piriou Y, Yang SC (2017) Effects of Polyphenolic Extract from Pine Bark on the Improvement of Attention Deficit/Hyperactivity Disorder in Children and Adolescent . Int J Clin Nutr Diet 3: 116. doi: https://doi.org/10.15344/2456-8171/2017/116

Page 2 of 4

\section{Study design}

It was a randomized, double-blind, crossover and placebocontrolled 10-weeks period study, including two interventional periods (4 weeks/period) and one washout period (2 weeks). During the first interventional period, participants were received a capsule of PE from pine bark, which contain $25 \mathrm{mg}$ Oligopin $^{\circledR}$ (Les Derives Resiniques Et Terpeniques, French) per capsule (Y), or a capsule of placebo (R), which contains $25 \mathrm{mg}$ cellulose. Then, participants entered the washout period for 2 weeks. After 2 weeks of washout, the participants entered the second interventional period and crossed over to receive an Oligopin $^{\circledR}$ or placebo capsule. Neuropsychological assessment, blood biochemical parameter and antioxidative status were determined at baseline and the end of every period.

\section{Blood biochemical parameters}

An autoanalyzer (SYNCHRON CX System, Hitachi 7170, Tokyo, Japan) was used to analyze blood biochemical parameters including liver function, kidney function, nutritional status, lipid profile, hematology and iron status.

\begin{tabular}{|c|c|c|c|}
\hline & Baseline & $\mathrm{R}$ & Y \\
\hline \multicolumn{4}{|l|}{ Physical measurement } \\
\hline Height $(\mathrm{cm})$ & $130.6 \pm 13.3$ & $131.6 \pm 13.1^{*}$ & $131.4 \pm 13.3^{*}$ \\
\hline Body weight (kg) & $29.9 \pm 7.5$ & $30.9 \pm 8.2^{*}$ & $31.4 \pm 7.1^{*}$ \\
\hline Body mass index $\left(\mathrm{kg} / \mathrm{m}^{2}\right)$ & $17.4 \pm 2.1$ & $17.6 \pm 2.2$ & $18.0 \pm 1.8^{*}$ \\
\hline \multicolumn{4}{|l|}{ Liver function } \\
\hline AST (U/L) & $28.14 \pm 3.18$ & $26.29 \pm 1.89$ & $28.57 \pm 3.87$ \\
\hline ALT (U/L) & $15.71 \pm 3.25$ & $15.14 \pm 2.48$ & $18.71 \pm 3.35$ \\
\hline Bilirubin-Total (mg/dL) & $0.59 \pm 0.11$ & $0.49 \pm 0.18$ & $0.37 \pm 0.14$ \\
\hline \multicolumn{4}{|l|}{ Kidney function } \\
\hline BUN (mg/dL) & $12.69 \pm 3.26$ & $12.69 \pm 2.98$ & $13.37 \pm 3.23$ \\
\hline Creatinine $(\mathrm{mg} / \mathrm{dL})$ & $0.48 \pm 0.06$ & $0.49 \pm 0.09$ & $0.46 \pm 0.07$ \\
\hline Uric acid (mg/dL) & $4.54 \pm 1.12$ & $4.63 \pm 1.12$ & $4.06 \pm 0.95$ \\
\hline Sodium (meq/L) & $140.71 \pm 1.25$ & $140.29 \pm 1.11$ & $139.86 \pm 1.35$ \\
\hline Potassium (meq/L) & $4.53 \pm 0.37$ & $4.44 \pm 0.26$ & $4.71 \pm 0.25$ \\
\hline \multicolumn{4}{|l|}{ Nutritional status } \\
\hline Albumin (g/dL) & $4.76 \pm 0.17$ & $4.89 \pm 0.25$ & $4.71 \pm 0.23$ \\
\hline \multicolumn{4}{|l|}{ Lipid profile } \\
\hline Triglyceride (mg/dL) & $57.86 \pm 26.43$ & $59.00 \pm 19.74$ & $66.43 \pm 36.29$ \\
\hline Cholesterol (mg/dL) & $171.14 \pm 16.43$ & $168.57 \pm 29.44$ & $165.00 \pm 22.23$ \\
\hline HDL-Cholesterol (mg/dL) & $59.14 \pm 9.48$ & $59.71 \pm 14.42$ & $60.14 \pm 12.25$ \\
\hline LDL- Cholesterol (mg/dL) & $100.43 \pm 14.74$ & $100.00 \pm 23.9$ & $93.57 \pm 13.04$ \\
\hline \multicolumn{4}{|l|}{ Hematology } \\
\hline WBC $(10 \mathrm{e} 3 / \mu \mathrm{L})$ & $6.90 \pm 2.56$ & $7.20 \pm 1.83$ & $7.17 \pm 1.44$ \\
\hline $\mathrm{RBC}(10 \mathrm{e} 6 / \mu \mathrm{L})$ & $5.09 \pm 0.74$ & $5.01 \pm 0.64$ & $5.03 \pm 0.76$ \\
\hline Hemoglobin (g/dL) & $13.50 \pm 1.23$ & $13.31 \pm 0.95$ & $13.26 \pm 1.27$ \\
\hline Platelet $(10 \mathrm{e} 3 / \mu \mathrm{L})$ & $343.86 \pm 44.7$ & $378.14 \pm 35.18$ & $367.00 \pm 72.94$ \\
\hline \multicolumn{4}{|l|}{ Iron status } \\
\hline Iron $(\mu \mathrm{g} / \mathrm{dL})$ & $118.29 \pm 40.49$ & $94.86 \pm 16.64$ & $72.00 \pm 23.01$ \\
\hline TIBC $(\mu \mathrm{g} / \mathrm{dL})$ & $374.29 \pm 35.95$ & $375.14 \pm 37.94$ & $376.14 \pm 29.23$ \\
\hline Ferritin (ng/mL) & $63.33 \pm 34.49$ & $48.89 \pm 23.77$ & $44.94 \pm 23.36$ \\
\hline \multicolumn{4}{|l|}{ Oxidative stress } \\
\hline TBARS $(\mu \mathrm{M})$ & $2.12 \pm 0.54$ & $2.11 \pm 0.48$ & $1.80 \pm 0.42^{*} \dagger$ \\
\hline
\end{tabular}

Table 1: Characteristics and clinical data of the placebo $(\mathrm{R})$ and intervention $(\mathrm{Y})$ groupsin participants with $\mathrm{ADHD}^{1}$.

${ }^{1}$ Values are expressed as the mean \pm SD.Data from 7 participants with attention deficit hyperactivity disorder (ADHD). ${ }^{*} \mathrm{p}$ $<0.05$ compared to baseline by pair t-test. $\dagger \mathrm{p}<0.05$ compared to $\mathrm{R}$ by pair $\mathrm{t}$-test.AST, aspartate aminotransferase; ALT, alanine aminotransferase; BUN, blood urea nitrogen; HDL, high density lipoprotein; LDL, low density lipoprotein; WBC, white blood cell; $\mathrm{RBC}$, red blood cell; TIBC, total iron binding capacity; TBARS, thiobarbituric acid reactive substances 
Citation: Chen YR, Su YJ, Piriou Y, Yang SC (2017) Effects of Polyphenolic Extract from Pine Bark on the Improvement of Attention Deficit/Hyperactivity Disorder in Children and Adolescent . Int J Clin Nutr Diet 3: 116. doi: https://doi.org/10.15344/2456-8171/2017/116

\begin{tabular}{|c|c|c|c|c|}
\hline & Concepts & Baseline & $\mathrm{R}$ & $\mathrm{Y}$ \\
\hline Inattention & & $\%$ & $\%$ & $\%$ \\
\hline Omissions & Accuracy/hit & $58.84 \pm 17.12$ & $57.68 \pm 16.56$ & $53.24 \pm 20.65$ \\
\hline Commissions & Accuracy/inhibition & $54.1 \pm 11.58$ & $50.72 \pm 10.17$ & $44.29 \pm 9.44^{*} \dagger$ \\
\hline Hit RT & Reaction time & $49.64 \pm 17.11$ & $52.08 \pm 16.10$ & $53.21 \pm 18.78$ \\
\hline Hit RT SE & Consistency/ sustainability & $51.7 \pm 12.36$ & $54.63 \pm 12.52$ & $50.55 \pm 12.72$ \\
\hline Variability & Consistency/ sustainability & $51.24 \pm 11.13$ & $52.37 \pm 11.69$ & $48.16 \pm 8.88$ \\
\hline Detectability & Accuracy/hit & $52.53 \pm 9.36$ & $52.21 \pm 9.00$ & $45.18 \pm 8.08 \dagger$ \\
\hline Hit RT ISI Change & Consistency/ sustainability & $52.97 \pm 6.26$ & $60.04 \pm 12.46$ & $55.59 \pm 4.97$ \\
\hline Hit SE ISI Change & Consistency/ sustainability & $48.67 \pm 13.65$ & $55.11 \pm 8.03$ & $49.44 \pm 5.18 \dagger$ \\
\hline \multicolumn{5}{|l|}{ Impulsivity } \\
\hline Commissions & Accuracy/inhibition & $54.1 \pm 11.58$ & $50.72 \pm 10.17$ & $44.29 \pm 9.44^{*} \dagger$ \\
\hline Hit RT & Reaction time & $49.64 \pm 17.11$ & $52.08 \pm 16.1$ & $53.21 \pm 18.78$ \\
\hline Perseverations & Accuracy/inhibition & $48.09 \pm 6.74$ & $51.94 \pm 8.82$ & $52.02 \pm 21.6$ \\
\hline \multicolumn{5}{|l|}{ Vigilance } \\
\hline Hit RT Block Change & Consistency/ sustainability & $52.28 \pm 10.92$ & $51.55 \pm 13.38$ & $49.35 \pm 10.59$ \\
\hline Hit SE Block Change & Consistency/ sustainability & $48.05 \pm 13.68$ & $46.14 \pm 10.08$ & $48.48 \pm 7.45$ \\
\hline
\end{tabular}

Table 2 The Conners' ContinuousPerformance Test (CPT-II) of the placebo (R) and intervention (Y) groupsin participants with ADHD1

1 Values are expressed as the mean \pm SD.Data from 7 participants with attention deficit hyperactivity disorder $(\mathrm{ADHD}) .{ }^{*} \mathrm{p}<0.05$ compared to baseline by pair t-test. $\dagger p<0.05$ compared to R by pair t-test. RT, reaction time; ISI, inter-stimulus interval; SE, standard error

\section{Antioxidative status}

Plasma thiobarbituric acid-reactive substance (TBARS) concentration was used as the lipid peroxidation indicator, which was quantitatively measured the TBARS concentration in plasma by the method of Ohkawa et al. [6].

\section{Neuropsychological assessment}

Neuropsychological test was assessed by psychologists. Conners' Continuous Performance Test (CPT-II) was used to evaluate the inattention, impulsivity and vigilance for children with ADHD.

\section{Statistical analysis}

All data are expressed as the mean \pm standard deviation (SD). Pair t-test was used to compare the baseline values of the groups (PE versus placebo) and also changes in each evaluated parameter during the course of the study between the groups using EXCEL software (Redmond, WA, USA). Statistical significance was assigned at the $\mathrm{p}<$ 0.05 level.

\section{Results}

Sixteen participants were recruited at first and 7 of them passed the screening test of SNAP-IV, CPM, SPM and CBCL. The characteristic of 7 participants ( 6 boys and 1 girl) was shown as Table 1 . The average age was $8.4 \pm 2.1$. The heights and body weights of participants were significantly increased after 10 weeks. However, all blood biochemical parameters didn't change during the experimental period and maintained in the normal range (Table 1). In addition, the lipid peroxidation was significantly decreased when participants received PE capsules (Y group) (Table 1). On the other hand, the result of neuropsychological test was shown in Table 2 . The variables may represent one or more clinical concepts at the same time, such as the data of commissions represents the concept of inattention and impulsivity. Compared with $\mathrm{R}$ group, participants had a significant lower commissions (accuracy/inhibition), detectability (accuracy/ hit) and Hit SE ISI change (consistency/sustainability) during the $\mathrm{PE}$ interventional period ( $\mathrm{Y}$ group). That is, the inattention and impulsivity were significantly improved when participants received PE capsules.

\section{Discussion}

This study demonstrated that polyphenolic extract from pine bark extract may have the potential to ameliorate the symptoms of ADHD in children. Simultaneously, the lipid peroxidation was also diminished when ADHD participants were supplemented by polyphenolic extract (PE) capsules.

The results reported by Tenenbaum et al. showed no treatment effect of PE extract on the ADHD symptoms of adults [7]. However, Trebatická et al. pointed to an option to use $\mathrm{PE}$ as a natural supplement $(1 \mathrm{mg} / \mathrm{kg} \mathrm{BW} /$ day) to relive ADHD symptoms of children [8]. The reasons for the inconsistent research findings are complicated, such as methodological factors of psychologicalmeasurements, age, ethnicity, and dosage of supplement etc. In this study, CPT-II which is the authorized assessment method for ADHD symptoms was used. Additionally, compared with the previous studies, the moderate dosage of PE (25 mg/day) was used in this study.

The mechanisms of intervention success remains to be elucidated. In this study, although PE reduced the oxidative stress by decreasing the plasma lipid peroxidation products in children with ADHD, more evidences supporting the link between oxidative stress and ADHD are necessary. 
Citation: Chen YR, Su YJ, Piriou Y, Yang SC (2017) Effects of Polyphenolic Extract from Pine Bark on the Improvement of Attention Deficit/Hyperactivity Disorder in Children and Adolescent . Int J Clin Nutr Diet 3: 116. doi: https://doi.org/10.15344/2456-8171/2017/116

\section{Conclusion}

In conclusion, the administration of the polyphenolic extract from pine bark $(25 \mathrm{mg} /$ day $)$ for one month might improve the inattention and impulsivity and reduce plasma lipid peroxidation levels in children and adolescent with ADHD.

\section{Conflict of Interest}

The authors declared no potential conflict of interest with respect to the research, authorships and publication of this study.

\section{Acknowledgement of Support and Assistance}

We thank Formosa Produce Corporation for providing the funds. We also appreciate Les Derives Resiniques Et Terpeniques for supplying the pine bark extract.

\section{Author Contributions}

Suh-Ching Yang designed the study. Yu-Ju Su and Ya-Ling Chen did the data analysis. The psychiatric evaluation was provided by Ying-Ru Chen, Wan-Lin Hsieh and I-Cheng Lin. Yannick Piriou provided the professional information and analyzed the composition of the pine bark extract.

\section{Funding}

This study was supported by the funds from Formosa Produce Corporation, Taipei, Taiwan.

\section{References}

1. Rowland AS, Lesesne CA, Abramowitz AJ (2002) The epidemiology of attention-deficit/hyperactivity disorder (ADHD): a public health view. Ment Retard Dev Disabil Res Rev 8: 162-170.

2. Tai YM,Chiu HW (2009) Comorbidity study of ADHD: Applying association rule mining (ARM) to National Health Insurance Database of Taiwan. Int J Med Inform78: e75-e83.

3. Wilens TE, Biederman J, Spencer TJ (2002) Attention deficit/hyperactivity disorder across lifespan. Annu Rev Med 53:113-131.

4. Joseph N,Zhang-James Y,Perl A,Faraone SV(2015) Oxidative stress and ADHD: a meta-analysis. J AttenDisord19: 915-924.

5. Heimann SW (1999) Pycnogenol for ADHD? J Am Acad Child. Adolesc Psychiatry 38:357-358.

6. Ohkawa H, Ohishi N, Yagi K (1979) Assay for lipid peroxides in animal tissues by thiobarbituric acid reaction. Anal Biochem 95: 351-358.

7. Tenenbaum S, Paull JC, Sparrow EP, Dodd DK ,Green L(2002) An experimental comparison of Pycnogenol and methylphenidate in adults with Attention-Deficit/Hyperactivity Disorder (ADHD). J Atten Disord 6: 4960.

8. Trebatická J,Kopasová S,Hradecná Z,Cinovský K,Skodácek I,et al. (2006) Treatment of ADHD with French maritime pine bark extract, Pycnogenol. Eur Child Adolesc Psychiatry15:329-335. 